Derecho \& Realidad

Núm. 24 • II semestre de 2014

Facultad de Derecho y Ciencias Sociales, UPTC

ISSN: 1692-3936

\title{
El profesor universitario en la era de la globalización*
}

\author{
Néstor Hernando Parra Escobar
}

Señor rector, profesoras y profesores amigos:

Hablar ante tan selecto auditorio constituye un honor y un compromiso que en gran medida es un reto, al menos para mí. Es también una excepcional ocasión para volver sobre un tema que ha ocupado mi atención en el campo de la educación superior: el profesor universitario. Sin embargo, prefiero comenzar por mis observaciones como estudiante universitario en cursos para mayores durante los tres últimos años, que me ha permitido constatar la alta formación y calidad de docentes profesionales, el desarrollo de la investigación, los programas de proyección social, la extensa gama de recursos físicos y tecnológicos, los nuevos métodos de trabajo en red, al igual que vestigios de metodologías docentes que suponía ya superadas, muestra clara de la resistencia ancestral de algunos profesores a cambiar su actitud verticalmente soberana frente a los educandos.

Reconozco el valor de las clases magistrales cuando de escuchar a maestros excepcionales, de eso se trata $-\mathrm{y}$ sí que los he tenido-, máxime cuando son complementadas con talleres de grupo; sin embargo, he visto a profesores que me han dejado la impresión de que van a oírse, mientras el alumno pasa a ser testigo mudo de que el maestro se está oyendo, con el resultado de que si bien la erudición del maestro genera admiración, en cambio no logra despertar suficiente motivación en el alumno, fundamento del aprendizaje. Motivación que en el proceso educativo involucra conocimientos y dimensiones vitales: valores, afectos, experiencias. Sin olvidar que hay quien se sienta en su trono y durante las dos horas de clase se limita

* Palabras del doctor Néstor Hernando Parra Escobar en la inauguración del semestre académico en la Universidad de Ibagué, Tolima, Colombia, el 1 de agosto de 2013. 
Derecho y Realidad

a alternar la lectura en voz alta entre anotaciones de un viejo cuaderno y apartes de un libro, por lo que el estudiante termina desertando, como fue mi caso. Todo esto, en una época en que la globalización, de la mano de la tecnología, ha puesto en gran parte la información y el conocimiento a libre disposición, sin consideración de lugar o tiempo, por cuanto el profesor y el alumno gozan del don de la ubicuidad mediante el impulso de un clic que los transporta al mundo cibernético de sistemas y subsistemas en red.

De allí que no resista a la tentación de hacer desde esta localización espacial y temporal -la Universidad de Ibagué, hoy-, un rápido sobrevuelo sobre el papel del profesor universitario en la era de la globalización, si bien ya hay quienes anuncian, como el filósofo canadiense John Ralston Saul, "El colapso de la globalización y la reinvención del mundo". Cabe recordar que desde el siglo pasado se viene afirmando que la misión principal del profesor ya no es la de enseñar, sino la de lograr que el alumno aprenda a aprender, proceso definitivamente interactivo y subjetivo, tanto si la clase es presencial o por cualesquiera de los medios de la educación digital (e-learning, blended-learning; mobile-learning), de evidente enriquecimiento colectivo, inclusive el del profesor.

Incita también a recordar la evolución de la educación en la historia de la humanidad, desde la Antigüedad, cuando los escribas, al servicio de la élite imperante, cumplían con la misión de transmitir sus tradiciones con base en sus mitos y la exaltación de la épica del respectivo grupo social; pasando luego por los monjes de conventos que en el largo Medioevo recordaban al ser humano su misión, la de alabar a Dios y esperar la muerte para encontrar el paraíso; hasta llegar al alba de la Modernidad en pleno Renacimiento con su visión antropocéntrica en la que el ser humano, guiado por el concepto de libertad que le conduce al ejercicio de su autonomía, pasa a ser protagonista de su destino en busca de su felicidad. Era en la que se reabre el apetito de conocer lo desconocido, se recrea conocimiento, se prioriza el interés en aprehender la realidad material antes que la verdad abstracta, se ambiciona dominar el mundo; surge el paradigma del progreso y comienza a ampliarse el ámbito de la educación a capas sociales ignoradas e ignorantes. Se ingresa en el camino infinito de la averiguación de lo desconocido ahora mediante la investigación científica, ya no como simple intuición del mundo de la percepción y la razón. Investigación que a la par que destruye mitos y erige nuevos principios y verdades descubre campos del saber que propician la desintegración de la ciencia y el surgimiento de especialidades que han adquirido la categoría de ciencias.

Fruto de ese continuo proceso de investigación científica que propicia dos revoluciones industriales y renovaciones de los sistemas de organización política y social, transitamos hoy por la Sociedad del Conocimiento basada en la proliferación de información que adquiere la categoría de valor social, por cuanto es determinante 
de nuevas pautas de comportamiento e indispensable en el mundo contemporáneo de las relaciones interpersonales en cuanto las redes virtuales permiten acceder a la comunicación instantánea entre los hasta ayer distantes y separados grupos sociales en todos los puntos cardinales desde donde acuden las nuevas generaciones ansiosas de reconocimiento. La información, cierta o falsa, logra penetrar en todas las sociedades sin importar regímenes, inclusive esquivando sistemas de control que intentan aislar a sus ciudadanos de la comunicación libre y universal, como lo analiza el sociólogo español Manuel Castells en el libro Las redes de indignación y esperanza, en relación con los movimientos recientes de protesta, desde los de Madrid hasta los de Wall Street, pasando por los de la "primavera árabe". A los que habría que agregar los muy frescos de Turquía y Brasil, de economías emergentes y perfil democrático, y la redición egipcia de su lucha libertaria.

Coincide el nacimiento de la Universidad de Ibagué con la entrada en firme de esta nueva época universal ya en plena Postmodernidad, que hace que lo real y lo virtual sean un continuum. Mundialización que agiganta el comercio internacional y de la mano de las tecnologías, destruye puestos de trabajo y crea nuevos, aún desabastecidos de personal, abarata la mano de obra y destruye fronteras nacionales para dar paso al capitalismo financiero global que transforma el dinero de generador de riqueza, en mercancía propia. Con este sistema acrecen las distancias de poder, se da pábulo a la codicia, se invade la órbita funcional del Estado y se minan valores éticos tradicionales. Joseph Stiglitz hablando de Estados Unidos en su libro El precio de la desigualdad, compara las abismales distancias entre el $1 \%$ más rico y el $99 \%$ más pobre en virtud del imperio del dinero y su impacto político, social y moral.

Es también el mundo en el que las habilidades humanas están siendo suplidas crecientemente por dispositivos creados por la inteligencia artificial, y se agiganta la brecha tecnológica entre naciones que generan tecnología y las que la pueden comprar para servirse de ella. Ante la abundancia de información "desordenada", que tiende a suplantar el verdadero conocimiento y la sabiduría se hace escasa, la conformación de un currículo de estudios superiores se torna en arte de magia, así como la asignación de las actividades del profesor en su ejecución. La magia de la imaginación propositiva.

Dentro de ese marco: cumplimiento de la misión de la universidad, avalancha liberadora del conocimiento, imperio de la economía del dinero, y la imaginación de una democracia cognitiva, ¿cuál el papel del profesor universitario en su maravillosa misión de educar y ayudar a formar a las nuevas generaciones en la era de la globalización? Tímidas aproximaciones, quizá más preguntas que respuestas, son las que intentaré realizar sin perder la localización espacial. 
Derecho ₹ Realidad

\section{Cumplimiento de la misión de la Universidad al compás de los tiempos}

Sin necesidad de hacer historia de estos más de sesenta semestres académicos ya transitados, ni de hacer comparaciones entre el primero y el que hoy se inicia, lo que además sería apasionante, es fácil reconocer que la Universidad ha estado avanzando armónicamente propulsada por la misión que trazamos los fundadores: promover la formación integral de líderes y empresarios con sólida formación científica y profesional, con arraigados principios éticos y morales, y comprometidos con el desarrollo social, cultural y económico regional. Esto ha sido posible gracias a directivas y profesores que han sido conscientes del vertiginoso ritmo de los cambios de la ciencia y la tecnología, de sus incuestionables efectos en la conducta humana y del valor agregado al desarrollo de la comunidad local y regional, factores clave para determinar la oferta responsable de educación superior en las diferentes áreas del conocimiento.

Merece especial mención la tarea que viene cumpliendo la Universidad, gracias a su probado poder de convocatoria, en el desarrollo social, mediante el fortalecimiento de políticas y programas en todo el departamento, entre los cuales destacan: el de Responsabilidad Social Integral, diseñado y puesto en ejecución por el Rector Reyes Alvarado, Victoria Kairuz y su grupo de trabajo; el programa pionero Apoyo a los municipios en la Reforma Descentralista, iniciado en la rectoría de Carmen Inés Cruz; el altamente valioso Paz y Región, hoy en plena acción y evaluación permanente, mediante el cual los estudiantes de último semestre de carrera viven en los municipios con el fin de brindarles asesoría, bajo la dirección y supervisión de docentes, y con base en su proyecto elaboran los respectivos trabajos de grado; y el programa Tolima 2025, en buena hora propuesto y reactivado recientemente por Eduardo Aldana Valdés en el que la institución ejerce liderazgo creativo. Todos ellos líderes universitarios consagrados al servicio del Tolima y a la exaltación de los tolimenses y sus valores, históricos y contemporáneos.

Siendo en gran parte la educación superior transmisión de información, sometida al tamiz del pensamiento crítico, base del proceso racional que genera conocimiento, las TIC también se ofrecen como vías igualitarias para pasar de la era artesanal, de pequeña producción presencial y acceso preferencial de las élites, a la de gran escala y democrática, a la educación digital. Ante tal paradigma, las nuevas metodologías se desempeñan como medios que facilitan a la par el trabajo independiente con la interactividad interdisciplinaria de profesores y alumnos a través de trabajos de redes que estimulan la conectividad y el enriquecimiento de las "Buenas Ideas" dando paso a la innovación y a la creatividad, que invaden las diferentes órbitas sociales y políticas hasta permitir imaginar el Futuro Perfecto que Steve Johnson describe en su reciente libro. Línea de desarrollo educativo que la Universidad de Ibagué tiene en plena actividad a través del Centro de Innovación 
Educativa Ávaco, con participación abierta a otras instituciones y en prometedora expansión con fines docentes e investigativos, en cuanto también estimula y hace posible el trabajo en red de investigadores de diferentes latitudes del mundo, ofreciéndose la democratización cognitiva sin fronteras.

Todo lo anterior, sin olvidar que la política que de tiempo atrás desarrolla la Universidad para formación y especialización de profesores a nivel de posgrado en instituciones nacionales y extranjeras registra notorios avances. En pocos años, la mayoría de los docentes serán doctores en su respectiva área de trabajo académico con lo que se tiende a garantizar mejor calidad en la educación que aquí se imparte.

En síntesis, la Universidad de Ibagué, con el continuo aporte de fundadores, benefactores, directivos, profesores y estudiantes, y la acogida del Estado y de la sociedad en general, se ha ido convirtiendo de establecimiento de educación superior en una institución social formal, por cuanto trasciende las funciones docentes e investigativas que cumple al compás de los avances científicos y tecnológicos globales. Institución humana, como todas, si bien, dada su filosofía, cabría mejor calificarla de Institución Humanista, es decir, al servicio de la perfección del ser humano como individuo y como miembro del conjunto social dentro de un amplio contexto espacial inclusivo, creando organizaciones apropiadas a sus fines de dignificación y superación del ser humano, regidos por códigos de comportamiento ético.

\section{La avalancha liberadora del conocimiento}

A comienzos de los sesenta, participé durante tres años consecutivos en seminarios de educación superior en la Universidad de Texas, de un mes de duración cada uno, justo cuando Estados Unidos vivía el esplendor de la política y programas de incentivación de la educación y particularmente de la investigación científica, como respuesta a la competencia por la conquista del espacio con la Unión Soviética, cuando desde el Cosmódromo de Baikonur, en 1957, se lanzó el sputnik, primer satélite artificial, y un mes después a la perra Laika, primer ser viviente en orbitar la tierra. En busca de ese florecimiento, las universidades estadounidenses asignaron al profesor la obligación de contribuir con sus investigaciones, sus logros y sus reflexiones críticas a ganar la partida del conocimiento científico universal, que expresaron en la máxima de to publish or to perish.

Los colombianos hacemos parte de los pueblos que luchan legítimamente, en esta época única como ninguna anterior, por hacer efectivo el derecho de participar y disfrutar de los avances de la civilización universal en procura de vida digna, en momentos en que se progresa simultáneamente en la aplicación de las TIC en las diferentes actividades humanas y en la revelación de lo Incógnito, la vida secreta 
del cerebro, su estructura y sus funciones, como los describe el neurocientífico David Eagleman, que sacuden creencias y conocimientos a la par que abren nuevos caminos de experimentación en diferentes áreas, entre ellas la de la educación, y muestran sus potencialidades así como sus limitaciones, como lo describe Francisco Mora, catedrático de Fisiología Humana, en su muy reciente libre Neuroeducación, la que define como "una nueva visión de la enseñanza basada en el cerebro. Visión que ha nacido de esta revolución cultural que ha venido en llamarse neurocultura".

Este veloz avance científico ha conducido el desgaje de las ciencias e inclusive al desconocimiento de la filosofía como "la ciencia", que ahora se propone remplazar por la "neurociencia" que se explaya a muy diversos campos del saber tal y como lo analizan profesores europeos y estadounidenses en el reciente libro Guía de neurofilosofía práctica. Entre esos avances destacan los de la mecánica cuántica, la neurociencia, la biogenética, la ecología, la astronomía, las ciencias aeroespaciales, la informática, la telemática, la robótica, la nanotecnología. La incorporación de la tercera dimensión en la producción industrial anuncia la tercera revolución industrial, la producción de energías alternativas limpias -la solar, la geotérmica, la eólica, la del hidrógeno y el nitrógeno-, el teletransporte, el conocimiento del genoma individual en escasos 15 minutos, el mayor alcance en la resonancia magnética, el análisis del ADN que facilitará la predicción de enfermedades y medicamentos individualizados, los rayos laser y sus múltiples aplicaciones, la modificación genética de alimentos y otros productos agrícolas. La telemedicina, los libros electrónicos y la educación digital son ya realidades, así como el poder destructor de las armas ubicuas de máxima precisión, sin dejar de lado el acceso a todo tipo de comunicación privada entre personas, el teleespionaje. Poder científico y tecnológico que unido al militar sigue aún concentrado mayoritariamente en Estados Unidos de América.

Es evidente que estos avances inciden en el ser humano, inclusive en la prolongación de la vida hasta tal punto que se anuncia que ya anda entre nosotros quien vivirá 400 años. En contraste, el de las ciencias no exactas, las sociales y las del comportamiento humano no han ido par y passu, con la salvedad de que las neurociencias que intentan explicar conductas y relaciones sociales y los avances en salud estén cambiando el temor a la muerte del que se han alimentado la mayoría de las religiones. El contraste se agranda al confirmar la irresponsabilidad con que se destruye el medio ambiente y la falta de conciencia y voluntad política para detener su camino hacia la hecatombe.

Ante la magnitud de los cambios que empiezan por la destrucción de conceptos, creencias, verdades y conocimientos que pierden validez, y también de derechos, como los de la privacidad y la intimidad, aflora la necesidad de innovar, de volver a inventar principios y valores -la solidaridad y la legitimidad entre otros-, de intentar armonizar ciencia y conciencia, progreso y naturaleza, riqueza e igualdad, 
cercanos y prójimos, con miras a seguir facilitando el ascenso del ser humano en su calidad de forjador de culturas en su proceso civilizatorio.

Es en ese escenario de fuerzas contrapuestas, de conciencia inteligente y de negación consciente en el que se mueve el ser humano, el educador tiene que interactuar con otros actores en una obra en la que si bien no está escrito su propio guion sí tiene que cumplir con la misión dignificante que le corresponde en cuanto al destino del ser humano. En el mundo incierto y contradictorio de hoy, el profesor parece estar obligado a colaborar en el proceso de creación colectiva tal y como lo hacen los actores en el happening o los músicos de jazz, por lo que tiene que innovar $o$ desaparecer. Como bien lo dijo Greth Mills en El Global Education Forum de 2010, los profesores deben convertirse en arquitectos del conocimiento, no en carteros. Innovar presupone una mentalidad abierta al cambio que es preciso adoptar, practicar y contagiar a los educandos, sin perder el telos.

Atrás quedaron los siglos de conservadora estabilidad. En el proceso de innovación, de transformación evolutiva, se induce a cambiar con la intención de acertar por lo que es menester utilizar métodos, como el bien conocido de ensayo y error, en contraposición al de la parálisis o inacción que también tiene sus riesgos y costos, que cumplan con la doble función de avanzar corrigiendo equivocaciones. Respecto del cambio, hay que subrayar que la actitud del profesor debe ir más allá de asumir los cambios, por cuanto implica la obligación de analizarlos mediante un estricto proceso de pensamiento crítico, analítico y creativo con el fin de adaptarlos o superarlos si fuere el caso.

Cambios en la metodología de la educación que también deben hacerse en forma experimental, en el más riguroso sentido científico, en los que hay que combinar medios tecnológicos como el internet fijo y el móvil, los videos, la "gamificación" o enseñanza mediante juegos interactivos, el "podcasting" o difusión de archivos de audio y video desde internet, el cine y las videoconferencias interactivas. La incorporación de estos medios, y de manera especial el tablero digital interactivo, ya en funcionamiento en una docena de aulas nuestra Universidad, cambia los escenarios educativos. Cambios a veces sencillos como el que se ha dado, por ejemplo, en el orden programado en la transmisión del conocimiento, comenzando por el último avance fundamental en la respectiva ciencia, dejando todo lo anterior como fruto del proceso histórico que también es necesario conocer y analizar.

Al profesor siempre se le ha exigido estar actualizado, pero el ritmo de los últimos años ha sido frenético: obligado a maniobrar las tecnologías de la información y la comunicación y a ser creativo en cuanto a su utilización como herramientas en el día a día de su trabajo. A partir de la mitad del siglo pasado, ha tenido que superar la clase discursiva, asimilando lentamente los aparatos audiovisuales y más 
rápidamente el computador y los demás medios, acostumbrándose a una realidad virtual. Fue así como los aspectos culturales y los informacionales de la globalización pasaron a influir directamente en el desempeño de su actividad central. Se vio obligado a enseñar virtualmente y a participar en las investigaciones colectivas en colaboración con colegas a quienes no conoce personalmente sino a través de la red. Y también a saber diferenciar el texto realmente elaborado por el alumno, del plagio mediante el simple procedimiento de "copia y pega" que el buscador de internet pone a disposición en su ofrecimiento de millares de referencias, siendo Google Académico su mayor expresión. Sin desconocer que eventualmente el alumno pueda poseer más conocimientos que él en determinado tema, comenzando por el manejo mismo de los nuevos dispositivos de las TIC.

\section{La dictadura de la economía del dinero}

Si algo ha cambiado drásticamente en las inexactas ciencias sociales es el orden jerárquico de la economía en el ordenamiento social. De economía política, al servicio del ser humano, se ha pasado a la economía en la que el objetivo es abstracto, numérico. Ciencias económicas que sirven más para analizar el pasado que para predecir el futuro en cuyo intento los economistas han tenido sonoros fracasos, como se ha demostrado en la actual crisis en Estados Unidos y Europa. Sin embargo, su importancia es cada día mayor en una época de desenfrenado consumismo y en el que la oferta excede la demanda con lo que se garantiza bajos precios que ayudan a incentivar el consumo.

La responsabilidad rectora de la economía, otrora en cabeza del Estado, ahora está en gran parte en "la mano invisible del mercado", encargada de asignar los recursos monetarios, inclusive los relacionados con la supuesta corrección de las carencias que genera en los grupos sociales discriminados. Ahora la máxima ganancia no es la humana o social, sino la del lucro del capital, del que poco o nada interesa quién se quede con la mayor parte de la torta. Recuerdo que hace tres años, en plena crisis, los estudiantes de economía de la Universidad de Harvard elevaron una protesta ante sus directivas exigiendo se les diera a conocer sistemas, escuelas, modelos económicos diferentes al del capitalismo financiero hoy imperante. Coincidían en cierta forma con defensores del sistema que hablan de la necesidad de armonizar crecimiento con igualdad y la de buscar modelos de consenso entre Estado y Mercado, indicando a los países escandinavos como referencia universal en cuanto exhiben altos índices de crecimiento económico y social, niveles mínimos de conflictividad, y sistemas educativos de máximo reconocimiento en el concierto mundial de naciones, con lo que se ratifica el papel que juega la educación en el gran conjunto social y político.

Queda por seguir explorando la alternativa teórica al neoliberalismo que hoy sirve de soporte a la globalización, a fin de dar forma a un sistema en el que prevalezcan 
valores morales más cercanos a lo bueno y lo justo, en el que mediante la extensión del conocimiento surja, se desarrolle y afinque la democracia del conocimiento, al servicio de todos los seres humanos y como partícipes del dinámico proceso de construcción de la democracia real. Lo cierto es que los científicos sociales se han estancado en la búsqueda de alternativas, limitándose unos a la defensa de la teoría de la globalización neoliberal y otros simplemente a denunciar sus estragos sociales.

En ejercicio de la independencia de los medios alternativos de comunicación y de algunos convencionales ha quedado en evidencia el imperio generalizado de la corrupción entre los miembros de las diferentes élites de poder, desde el político y el militar hasta el religioso. El afán de enriquecimiento rápido ha invadido inclusive campos en los que en forma despiadada se lucran de dineros públicos, o se los roban. Lepra social producto de la confusión ética intencionada entre las esferas de lo público y lo privado, que es preciso denunciar y combatir como factor social perverso, por cuanto ha llegado a gozar de cierto grado de indiferencia y de callada complicidad de los ciudadanos, aunque ya hastiados comienzan a hacerse ver y oír masivamente en España y Brasil.

Más que crecimiento lo que se requiere es desarrollo, progreso, como el opuesto en la díada de pobreza; progreso que hay que relacionarlo con democracia a fin de conocer su reflejo en cuanto a expresión de pluralismo político; la pobreza, en cambio, es menester observarla en función de bienestar humano y no de la riqueza monetaria. De lo que se trata es de intentar eliminar o por lo menos reducir al máximo y castigar de forma ejemplar los delitos de corrupción y poner fin a la codicia de los dueños de capital.

\section{Imaginando la democratización cognitiva}

En el proceso de innovación, la primera pregunta que aflora a la mente es qué deberíamos conservar y qué deberíamos destruir. Pensadores contemporáneos resaltan que en esta época de veloces cambios tan lentamente asimilados por las sociedades de humanes, hay mucho por aprender y también por desaprender, es decir, de abandonar ciertos memes "rasgos culturales o unidades de cultura también llamados ideas o símbolos," como los define Jesús de Mosterín en su libro La cultura humana, impuestos por el entorno en que se nace y se crece. "Ideas culturales" producto de la interacción simultánea de diversas fuerzas sociales, económicas y políticas en un determinado medio que llegan a constituir subculturas que, inconsciente o resignadamente, adoptan los individuos: la subcultura de la violencia, la de la corrupción, la de la desigualdad y la de la pobreza generalizadas en el último medio siglo en Colombia entre amplios sectores de la sociedad, tal y como lo imaginamos hace tres años aquí en la tertulia Cultura y Desarrollo en el marco de la celebración de los 30 años de fundación de la Universidad cuando 
Derecho ₹ Realidad

hicimos un recorrido de la historia contemporánea del Tolima: 60 años ya vividos a partir de 1950, período que denominamos el conflicto, y los 40 años por vivir que llamamos el postconflicto.

En ese mapa de navegación temporal, señalamos la posibilidad incorporar a la mujer y a la universidad colombiana como nuevos actores sociales que, junto con los convencionales, ayuden a urdir tejidos sociales frescos de los cuales surja la cohesión social como ejercicio real del principio de solidaridad. Y asignamos al profesor universitario la conducción responsable de sus alumnos en el proceso educativo, los reales actores sociales del futuro, avivando en ellos la sed y el hambre por el conocimiento que deben satisfacer no con los tradicionales medios, maestro, tablero y libro, sino mediante el uso de los que potencian el estudio independiente, la autodisciplina, la concentración y la solidaridad social. Destacamos la necesidad de fortalecer en el profesor competencias comunicativas y metodológicas en busca de la máxima eficiencia y productividad social. Y, por sobre todo, educar en pensamiento crítico y en valores.

Cuando se habla de pensamiento crítico, es preciso comenzar por aplicarlo a la incesante avalancha tecnológica que con sus artilugios engancha al ser humano e ingenuamente se somete a su esclavitud llevándolo a adoptar conductas zombi ante los atractivos de interrelación que hoy ofrecen las telecomunicaciones. Neil Postman, uno de los sociólogos y educadores que mejor ha analizado los efectos que la sociedad tiene que asumir ante los avances tecnológicos, los resume en su libro Technopoly. The surrender of culture to technology en el menoscabo de la cultura, su inequitativa distribución entre los diferentes segmentos sociales, las consecuencias físicas y emocionales en el ser humano, el poder de destrucción sobre todo lo pre existente y la tendencia a convertirlas en mito. Cabría añadir la explotación económica que los propietarios de las respectivas patentes realizan con el fin que les es propio, el máximo lucro.

Tales consecuencias sociales y culturales no deben servir de obstáculo para reconocer su utilidad en cuanto se usen como lo que son, medios, cuyo uso inteligente puede servir para avanzar en el logro de los fines superiores del espíritu en la lucha que, desde Prometeo, realiza el hombre por desencadenarse de la finitud en busca de alcanzar el sueño de la infinitud.

En este camino, un tanto caótico de la sociedad de hoy, la ética de la solidaridad juega papel básico en la actividad del profesor, ética extendida a través de todo el proceso, bien distinta del moralismo con el que se manipula impunemente. Solidaridad universal basada en los principios de libertad e igualdad que respete la plétora de las culturas y que se oriente hacia el surgimiento de un nuevo humanismo, 
en el que estado y sociedad, política y economía, neurociencia y libre albedrío coexistan de forma creativa.

Educar en la construcción de un nuevo humanismo en el que concilie ciencia y conciencia, sin confundir información con conocimiento y muchísimo menos con sabiduría; propiciar la cooperación antes que la competencia entre los educandos; ayudar a liberar al ciudadano de la dictadura consumista de la economía imperante; alimentar el apetito por el saber y el hábito del estudio permanente; infundir el respeto por las leyes en remplazo del sometimiento a los dogmas; prepararlos para el ejercicio de la democracia participativa y la democracia deliberativa, dialógica, en lugar de la representativa que está sucumbiendo ante el autismo y la corrupción de la clase política y los partidos políticos; valorar a la mujer llamada a jugar papel fundamental en la ciencia y en la convivencia y a convertirse en la heroína de la libertad en culturas donde aún las excluyen y discriminan en la vigencia de los derechos humanos; colaborar a la reducción de la desigualdad social.

Educar en el amor y el respeto por la naturaleza de la cual su fruto dorado es el ser humano, antes que explotarla y destruirla irresponsablemente, cuyos efectos en el medio ambiente son cada día más alarmantes. La desatención de gobiernos, políticos, empresarios y ciudadanos en general al tema ecológico no puede continuar en momentos en que prevalece la explotación criminal de sus recursos por quienes solo les interesa el lucro; parar la destrucción inmisericorde del aire y del agua, elementos básicos de la vida. Adela Cortina, la reconocida filósofa valenciana, en su último libro ¿Para qué sirve realmente? La Ética, propone que el biocentrismo sustituya el antropocentrismo por cuanto es preciso abordar los problemas de la naturaleza global, y no de manera unilateral como lo hace el pensar tecnológico, porque hay interdependencia entre todos los lugares del planeta" lo que hace "preciso promover el < yo ecológico > de las personas, y no solo el < yo social >; y es necesario crear la comunidad biótica, no sólo la comunidad política". A esto cabría agregar por nuestra cuenta que en esa comunidad biótica no imperaría la ética de la supervivencia individual, ni siquiera la de la convivencia pacífica entre los mismos, sino la ética de la coexistencia con la naturaleza tendiente a garantizar la supervivencia de la especie y la convivencia entre todos.

Educar para desarrollar en el educando su capacidad de investigación, innovación y emprendimiento -no simplemente la de empresario-, con un generoso criterio colectivo, por cuanto el empleo de hoy seguirá siendo objeto de destrucción por las aplicaciones tecnológicas, las economías basadas en el lucro y gobiernos desentendidos de su función social. Empleo cada año más esquivo para las nuevas generaciones de egresados universitarios y que constituye uno de los campos en que mayor imaginación profética deben aplicar directivos y profesores encargados 
Derecho y Realidad

del diseño de los programas profesionales o formativos para facilitar el objetivo de realización personal y contribución social del egresado, así como la obtención del ingreso que le permita llevar una vida decorosa en el conjunto social. Emprendedor, empresario, auto empleado y empleado, presencial o a distancia, son roles en los cuales el nuevo profesional debe saber desenvolverse en épocas de acelerada destrucción creativa, y que el currículo debe prever. Educar no sólo para el bienestar, para el bienvivir, sino fundamentalmente para el "bienser", para que el humano busque su felicidad mediante la armonía con sus demás congéneres y con la naturaleza.

Apasionante tarea la del profesor universitario, y la del profesorado como colectivo interactuante y creador, la de imaginar el futuro en forma amplia e inclusiva en un nuevo contexto cultural humanista, sirviéndose de las ciencias y las tecnologías; la de ayudar a forjarlo mediante la práctica de la observación, la investigación, la innovación. el pensamiento crítico y la vigencia ética de los valores, más allá de la visión utilitarista que hoy impera; la de romper las finitudes, a sabiendas de que se crean nuevas, desencadenando las fuerzas liberadoras del espíritu hacia fines de convivencia y de armonía con el universo; he ahí la esplendidez de su noble misión. Pero, ¿cuáles serán sus nuevas funciones en el proceso educativo? ¿Acaso será mayor la de coaching que la de transmisor de información y conocimientos? ¿Tomará mayor importancia la de tutor que la de profesor, la de certificador por encima de la de instructor? ¿Cómo desarrollar las competencias de comunicación en un universo de interconectividad?

A este último interrogante, el joven filósofo valenciano Francisco Arenas-Dolz responde señalando algunos beneficios del uso del internet en el proceso educativo: 1. Entraña el desarrollo de una cultura educativa. 2. Asume la hibridación de los entornos educativos. 3. Crea modelos pedagógicos que permiten el aprovechamiento cognitivo distribuido en red. 4. Amplía la educación formal a procesos no formales e informales, lo que supone "descontracturar" el aprendizaje. 5. Añade a la relación entre alumnos en red el beneficio de la cooperación. Como puede apreciarse lo que se vislumbra es que el profesor de la era de la globalización es y será distinto al que hasta ayer conocimos.

Este sobrevuelo nos confirma la complejidad del mundo en el que vivimos y la necesidad de buscar, al igual que respuestas prácticas, una síntesis, un pensamiento unificador que abarque todas las partes, particularmente aplicable al papel del profesor en plena era de la globalización. Me aventuro a proponer que ese pensamiento integrador sea el de la armonía, expresión de equilibrio, de acuerdos, de consensos, de conjunción de sinergias. Armonía entre ciencia y filosofía, entre tecnología y ser humano, entre naturaleza y progreso, con la pretensión de que sus logros tengan aceptación y validez universal. 
Amigas y amigos docentes:

Tienen ustedes la enorme ventaja de pertenecer a los mundos maravillosos de la realidad del aquí y ahora, y al imaginario del futuro, unida a la facultad de ayudar a forjarlo. Están ustedes viviendo una de las épocas más apasionantes de la historia, caracterizada, como lo hemos visto, por los cambios veloces del conocimiento, de las ciencias y de las tecnologías, con sus correspondientes efectos en el campo del comportamiento humano, que en su conjunto constituyen retos que ustedes tienen que asumir críticamente para entenderlos, cuestionarlos, adaptarlos y, lo fundamental, saberlos compartir con sus educandos de tal forma que les sirvan para orientarlos en sus propias vidas, teniendo en cuenta el espacio territorial y el conjunto social en el que laboran, en los que si bien se vislumbran horizontes menos siniestros a los del inmediato pasado, son grandes las secuelas de las subculturas perversas que aún hay que ayudar a superar.

Señor rector:

Especial satisfacción y fundada esperanzas las que se sienten al observar el progreso de la educación superior en nuestro departamento: de los 100 del inicio de mi período de rector de la Universidad del Tolima, hace justo 52 años, hoy son cerca de 40 mil en 14 instituciones; de 2 programas académicos, hoy se ofrecen más de 220 debidamente calificados; de los primeros 14 graduados de 1962, hoy son más de $40 \mathrm{mil}$; la veintena de profesores de ayer se ha multiplicado en tal forma que excede los cuatro mil con diferentes grados de dedicación. Ejercicio aún más excitante al ver la evolución de la Universidad de Ibagué en sus 33 años de vida, cuya medición cuantitativa hay que multiplicar en beneficios sociales en virtud de la filosofía que los fundadores marcamos y que usted y sus compañeros de directivas interpretan en forma magistral.

Es usted experto conocedor del mundo de la ciencia y la tecnología y del quehacer universitario y posee virtudes que concitan voluntades a su alrededor. Comienza una nueva aventura por rumbos que, así parezcan conocidos y experimentados, tendrá su propia bitácora, travesía en la que contará con la eficiente cooperación y compañía de este responsable grupo de amigos y amigas docentes, garantías más que suficientes para seguir avanzando colectivamente con seguridad, optimismo y alegría en esta contractiva tarea de educar al servicio de la juventud y del desarrollo integral de la región. Noble y admirable tarea en la que estoy seguro seguirán cosechando éxitos altruistas.

Gracias 